\title{
Ejecución presupuestal y calidad de gasto en un gobierno local, periodo 2019
}

Budget execution and quality of spending in a local government, period 2019

Execução orçamentária e qualidade dos gastos em um governo local, período 2019

Carranza Isuiza, Víctor Benoni vcarranzai@ucv.virtual.edu.pe Universidad César Vallejo - Perú ORCID: 0000-0003-2462-9623

\author{
Rivero Tapullima, Leidi Laura \\ lriverot@ucv.virtual.edu.pe \\ Universidad César Vallejo - Perú \\ ORCID: 0000-0003-4833-9268 \\ Bernales Vasquez, Renan \\ rbernalesv@ucvvirtual.edu.pe \\ Universidad César Vallejo - Perú \\ ORCID : 0000-000-4189-9000
}

\author{
Villafuerte de la Cruz, Avelino \\ avillafuerte@ucv.edu.pe \\ Universidad César Vallejo - Perú \\ ORCID: 0000-0002-9447-8683
}

\section{RESUMEN}

El presente estudio de investigación tuvo como objetivo establecer la relación entre ejecución presupuestal con la calidad del gasto público en la Municipalidad Distrital de Shapaja, periodo 2019. Se justificó en aportes teóricos de autores que permitieron el desarrollo de la investigación, las bases teóricas que ayudaron a evaluar la problemática a través de los instrumentos de medición. La investigación fue de tipo básica, diseño no experimental, Se tuvo una población de los trabajadores de la municipalidad distrital de Shapaja con un total de 19 personas y el acervo documentario respecto a la ejecución presupuestal y calidad del gasto público (PIA y PIM). En cuanto a la técnica fue el análisis documental y la encuesta y el instrumento la guía del análisis documental y el cuestionario. Teniendo los siguientes resultados, la correlación de Pearson entre ejecución presupuestal y calidad de gasto de la Municipalidad Distrital de Shapaja, periodo 2019 es alta; con valor de (0.889) y la significancia bilateral menor a 0,05 (p<0,000).

Palabras claves: Ejecución presupuestal, calidad de gasto, gastos corrientes, categoría presupuestal, economía.

\begin{abstract}
The objective of this research study was to establish the relationship between budget execution and the quality of public spending in the District Municipality of Shapaja, period 2019. It was justified in theoretical contributions by authors that allowed the development of the research, the theoretical bases that helped to evaluate the problem through measurement instruments. The research was of a basic type, non-experimental design, there was a population of workers from the district municipality of Shapaja with a total of 19 people and the documentary collection regarding the budget execution and quality of public spending (PIA and PIM). Regarding the technique, it was the documentary analysis and the survey, and the instrument was the guide to the documentary analysis and the questionnaire. Having the following results, the Pearson correlation between budget execution and quality of spending of the District Municipality of Shapaja, period 2019 is high; with a value of (0.889) and the bilateral significance less than $0.05(\mathrm{p}<0.000)$.
\end{abstract}

Keywords: Budget execution, quality of spending, current expenses, budget category, economy.

\section{RESUMO}

A presente pesquisa teve como objetivo estabelecer a relação entre a execução orçamentária e a qualidade dos gastos públicos no Município Distrital de Shapaja, período 2019. Justificou-se nos aportes teóricos de autores que permitiram o desenvolvimento da pesquisa, as bases teóricas que auxiliaram a avaliar o problema por meio de instrumentos de medição. A pesquisa foi de tipo básico, de desenho não experimental, houve uma população de trabalhadores do distrito municipal de Shapaja com um total de 19 pessoas e o acervo documental referente à execução orçamentária e qualidade do gasto público (PIA e PIM). Quanto à técnica, fez-se a análise documental e o levantamento e o instrumento serviu de 
guia para a análise documental e o questionário. Tendo os seguintes resultados, a correlação de Pearson entre a execução orçamental e a qualidade da despesa do Município Distrital de Shapaja, período de 2019 é alta; com valor de $(0,889)$ e significância bilateral menor que $0,05(\mathrm{p}<0,000)$.

Palavras-chave: Execução orçamentária, qualidade dos gastos, despesas correntes, categoria orçamentária, economia.

\section{INTRODUCCIÓN}

El presupuesto público es el medio que se necesita para que el estado logre buenos efectos de mediación en dar calidad de los bienes y servicios, que estén de acorde al costo justo, eficiencia y que trata de ser efectivo y equitativo al ser brindado a sus usuarios y esto debería buscar mejorar las posibilidades. Esto viene a ser una de las primordiales ocupaciones del país, brindar el mejor producto y servicio público con la más alta calidad. Estas actividades ayudan a las personas a obtener bienestar y reducir la pobreza al promover el desarrollo de nuestros ciudadanos.

De acuerdo con la realidad problemática de la investigación, en el contexto internacional: Nicaragua viene reforzando su sistema presupuestario desde hace mucho tiempo y hoy por hoy está elaborando un marco presupuestario de mediano plazo (mpmp), que compone el marco institucional de gasto de mediano plazo (mimp), cuyas metas y objetivos son consistentes con el plan de progreso humano del país. El presupuesto tiene una organización programática, que incorpora productos finales, metas institucionales e indicadores (Engl et al, 2016).

A lo largo de los últimos 10 años, el costo del personal estatal se ha incrementado drásticamente. De acuerdo con el Ministerio de Economía y Finanzas (MEF), los salarios de los colaboradores de las entidades públicas se acrecentaron de S/ 18 mil millones a S/ 41 mil millones entre el año 2009 y 2018 . El incremento de este gasto hace que se disminuyan recursos usados para objetivos más productivos, como la inversión pública. Asimismo, del total de los gastos administrativos, el $31 \%$ pertenece a la inversión pública, lo que se asemeja a la compra de activos no financieros (El comercio, 2019).

La Municipalidad del Distrito de Shapaja es un ente del gobierno loca teniendo como función principal la administración de recursos públicos, adicionalmente busca el progreso de la población y cumplir con los intereses colectivos. Se encuentra situada en el caserío de Shapaja, capital del Distrito de Shapaja, inherente a la Provincia y Región de San Martín. De acuerdo al aplicativo del Ministerio de Economía y Finanzas (2021), consulta amigable, podemos interpretar que la entidad abarca problemas con la ejecución presupuestaria ya que su presupuesto inicial va modificándose de manera paulatina para asignar presupuesto a proyectos y actividades que se pretenden ejecutar. Del mismo modo, estos no llegaron a dar cumplimiento y satisfacción a las carencias primordiales de la población.

Adicionando a esto, que los gobiernos nacionales cuentan con gestiones deficientes para dar financiamiento a proyectos y obras de gran magnitud, acorde al aplicativo antes mencionado, se observa que solo se ejecutó el $37.7 \%$ del presupuesto asignado, siendo lo ideal ejecutar en su totalidad, igualmente se puede evidenciar que en el año 2018 ha tenido una mejor ejecución que en el año 2019, paralelo a eso, las actividades, acciones y programas que se han venido realizando por la entidad no se ha reflejado al momento de cubrir las necesidades de la ciudadanía.

Tabla 1

Ejecución presupuestal año 2018 y 2019

\begin{tabular}{llcl}
\hline Año & PIM & Ejecución & $\%$ \\
\hline 2018 & $2,610,594$ & $2,500,368$ & $95.8 \%$ \\
2019 & $6,182,267$ & $2,328,447$ & $37.7 \%$ \\
\hline
\end{tabular}

Nota. Datos tomados del aplicativo consulta amigable MEF.

En relación al problema general: ¿Qué relación existe entre ejecución presupuestal y calidad de gasto de la Municipalidad Distrital de Shapaja, periodo 2019? Del mismo modo, para profundizar en ellos se formuló los problemas específicos: ¿Qué relación existe entre gasto corriente y calidad del gasto de la Municipalidad Distrital de Shapaja, periodo 2019?, ¿Qué relación existe entre gasto de capital y calidad de gasto de la Municipalidad Distrital de Shapaja, periodo 2019?, ¿Qué relación existe entre categoría presupuestal y calidad de gasto de la Municipalidad Distrital de Shapaja, periodo 2019?

Por consiguiente, planteamos como objetivo general: Determinar la relación que existe entre ejecución presupuestal y calidad de gasto de la Municipalidad Distrital de Shapaja, periodo 2019. De la misma manera, los objetivos específicos: Determinar la relación del gasto corriente y calidad de gasto de la Municipalidad Distrital de Shapaja, periodo 2019. Determinar la relación de categoría presupuestal y calidad de gasto de la Municipalidad Distrital de Shapaja, periodo 2019. 
La ejecución presupuestaria municipal, incluida la gestión adecuada y eficaz de los recursos económicos, son funcionalidades del gobierno como agencia local, generalmente se formula en el plan de desarrollo coordinado, que incluye las precedencias de incremento socioeconómico de su territorio. Sin embargo, esta postura previsible no siempre es calificada como positiva por los habitantes, por ende; desde la perspectiva de la ciencia contable se sustenta teóricamente, ya que favorecerá a ofrecer información sobre las variables estudiadas. Desde el método desde la ciencia contable, se puede considerar la formulación de planes y otros proyectos basados en las teorías detalladas en la investigación. Desde la justificación práctica, permitirá comprender cómo se implementará el presupuesto municipal, para así determinar la relación causal que se da en el proceso contable y financiero, para aplicarlos desde el propio campo de la gestión pública para la reducción de la pobreza y exclusión colectica. La defensa de la investigación desde la esfera social, se fundamenta en que permitirá al gobierno municipal del Distrito de Shapaja mostrar la situación y problemas en la ejecución del presupuesto; ayudando a comprender el efecto de los resultados obtenidos y en los habitantes, lo que integra el fin de cualquier intervención estatal.

Por tanto, se planteó como hipótesis de investigación (Hi): La ejecución presupuestal se relaciona significativamente con la calidad de gasto de la Municipalidad Distrital de Shapaja, periodo 2019.

En la investigación se desarrolla en el apartado dos el fundamento teórico, en la que se muestra las investigaciones relacionadas a las variables en estudio, como también la base teórica de las variables y dimensiones, por otro lado en el apartado tres, se desarrolla los procedimientos metodológicos, donde se describe la metodología utilizada, las técnicas e instrumentos aplicados en la investigación, en el apartado cuatro, se desarrollan los resultados y discusiones, se presenta los resultados en forma narrativa y grafica de acuerdo a los objetivos planteados, se realiza la prueba de hipótesis, con un análisis crítico sobre las mismas, para posteriormente comparar los mismos con otros estudios similares, sigue el apartado cinco que son las conclusiones, se presenta en forma resumida la respuesta al objetivo del estudio, como se legró a lo que se llegó, también mostrar las limitaciones y sugerir futuras investigaciones, por último se presenta las referencias utilizadas en el desarrollo del artículo.

\section{FUNDAMENTO TEÓRICO}

\subsection{Antecedentes}

Referente a las investigaciones que anteceden, tenemos en el ámbito internacional a Barona López (2019), en su tesis de maestría "El plan operativo anual y la ejecución presupuestaria de las universidades y escuelas politécnicas públicas de la zona 3". Ecuador. Proponen evaluar el mencionado plan para brindar mejora a la gestión de la planificación institucional. Corresponde es exploratorio su tipo, diseñado de forma no empírica. El acervo documentario fueron su población y muestra. Resolviendo que las escuelas politécnicas y las universidades no contienen con métodos instaurados para ofrecer el cumplir de las ocupaciones que se han programado en su plan operativo anual (POA), incluso de disponer de las planificaciones rutinarios que de nada a poco ayuden a su control de forma idónea las ocupaciones y los presupuestos. Por consiguiente, se concluyó que hay la necesidad de reformar a la ejecución de métodos que se operan y que consigo ofrecen la manera de ejecución de los presupuestos.

Campoverde y Pincay (2019), en su artículo científico titulado "Ejecución presupuestaria y rendición de cuentas, sobre el rubro de investigación". Ecuador. Plantearon como objetivo abordar la realidad en una Universidad Ecuatoriana y su rol con la rendición de cuentas. El análisis fue de tipo documental cualitativa. Concluyendo que el sector público posee herramientas legales para cumplir su rol con la rendición de cuentas de esta manera se pueda llegar a conocer los logros y progresos de la administración pública, por medio de su organismo de gestión y control, contiene lineamientos documentales y formatos predeterminados para la difusión del presupuesto que ejecuta.

Desde otra perspectiva, Lemus et al. (2018), en su artículo científico denominado, "Análisis de la ejecución presupuestal en Colombia durante el periodo 1954-2013". Cuyo objetivo es reconocer la conducta de los desembolsos públicos de Colombia entre el año 1954 y 2013. Para este fin recurrió al método cuantitativo, particularmente a la identificación de modelo para serie temporal de Box-Jenkis. Lo cual posibilita detectar los cambios suscitados en los costos de inversión. Al final concluyeron que los desembolsos públicos se hallan en enorme medida repercutidos por los planes de desarrollo de cada régimen.

Toledo Gómez (2017), “Análisis de la Gestión en la ejecución presupuestaria en la Corporación Eléctrica Del Ecuador CELEC EP. Para la obtención de resultados plantea analizar la manera de gestionar la ejecución presupuestaria de la unidad de Negocio. Aplicando el método de investigación básica. La muestra estuvo anteriormente determinada en funcionalidad del objeto de análisis y el sitio de aplicación. La técnica e instrumento ha sido entrevista. Finiquita que se prueba en la carencia de administración para seguir los indicadores y la manera de tomar las acciones que se prevengan y corrijan de manera definidas y específicas.

Todesco y Cardoso Da Silva (2021), "Planificación sectorial y ejecución presupuestaria en turismo en Brasil (2003-2018)". Al enfocarse en la asignación y ejecución presupuestaria del MTur, se verifica que desde 2003en 2006 la carpeta pasó por un lapso de fortalecimiento y ocupó una postura de relativa destacó en la agenda 
de políticas de régimen en el lapso 2007 a 2010, una vez que presentó crecimiento constante tanto en la dotación como en la ejecución de recursos, concentrado principalmente en la zona de infraestructura. De 2011 a 2018 , el turismo ocupó un puesto periférico en la agenda del gobierno, lapso en el cual el MTur muestra una alta inestabilidad. Presupuesto, con contingencia significativa, escenario que indica inconvenientes en la composición de su presupuesto, así como en la función de administración del MTur en ejecución de programas y proyectos.

Viante et al. (2021). "Ejecución presupuestaria en Santa Catarina: Evolución del gasto de 2006 a 2018 " Los costos son elementos fundamentales de la gestión pública, debido a que están sujetas a los recursos que se gastan, puede haber interferencia en el desarrollo de la sociedad, siendo fundamental para la toma de elecciones de parte de burócratas gubernamentales, para mejorar la administración del gasto público y direccionar en qué superficies se tienen que ejercer los recursos. El presente trabajo tiene por objetivo examinar la evolución de los costos, por conjuntos de naturaleza, en el Estado de Santa Catarina, en el lapso de 2006 a 2018.

En un ámbito nacional tenemos a, Chafloque Farfán (2017) en su investigación titulada, "El presupuesto por resultados y sus efectos en la calidad de gasto del sector salud de la Región Lambayeque 2011 - 2016". A partir de un enfoque cuantitativo, con diseño no experimental y transversal. Teniendo como finalidad decidir los efectos del presupuesto por resultados ante la calidad del gasto de dicho sector. Mostrando que la población se beneficia por los diversos programas presupuestales (PpR) y que al fin y al cabo la ejecución del gasto logra cumplir con las metas propuestas.

Luna De Los Ríos (2016) en la investigación “La evaluación por calidad del gasto y su incidencia en la adecuada ejecución presupuestaria en Dirección Regional de Salud- Cajamarca, periodo 2014”. El autor realiza el estudio con el objetivo de implantar que la exploración de la calidad de gasto influye positivamente en una más grande revisión de la ejecución de presupuestos. El diseño es no experimental. Concluyendo de esta manera que los gastos incorporados en el presupuesto han sido debidamente invertidos y han tenido un excelente impacto en las metas específicas del plan y en las condiciones para normalizar los gastos de adquisiciones de los distintos organismos de acuerdo con las metas que logre.

De tal modo, Olivera Lucano (2018), en su tesis "Ejecución presupuestal de inversiones y su influencia en el alcance de objetivos del Municipio Provincial de Cutervo". Diseño de indagación no experimental. El análisis documental fue utilizado como instrumento. Concluye midiendo el logro de metas programadas del efectivo colectivo del año 2017, determinó que el 70\% se realiza en los campos de salud, electrificación, saneamiento, educación y transporte con una ejecución de S/ 8,348,161.00 homólogamente del $65.5 \%$ de lo programado en esta meta.

Paco y Mantari (2014) En la pesquisa denominada, "El proceso de ejecución presupuestal y su influencia en la calidad de gasto de la municipalidad provincial de Huancavelica periodo 2013". La finalidad del trabajo condujo a la evaluación de medida el proceso de la ejecución presupuestaria afecta la calidad de los gastos de la entidad. Llega a conclusión de que una ejecución correcta del presupuesto mejorará la calidad de los gastos y la utilizará para mejorar el nivel de vida de los habitantes.

\subsection{Teorías relacionadas}

En cuanto a las teorías relacionadas a la primera variable. Sostiene Santiago Chávez (2018) La ejecución presupuestaria es el inicio de las acciones que tienen como objetivo optimizar los recursos disponibles para obtener bienes y servicios que puedan lograr objetivos. Esta etapa incluye la asignación de recursos con base en el tope presupuestario aprobado, la ejecución de actividades diseñadas para cumplir con el plan aprobado con base en los gastos acumulados del presupuesto aceptado y la presentación periódica de herramientas de informes de ejecución. (pp. 22-23), mientras que Morales et al. (2018), indica: "Se da por las instituciones del sector público, y se realiza para adquirir bienes y servicio con la intención de cubrir las penurias del pueblo y contribuir a las riquezas estatales" (p.1).

De igual manera de acuerdo al MEF (2020), la Directiva $\mathrm{N}^{\circ}$ 007-2020-50.01 - Directiva para la ejecución presupuestaria. Menciona las siguientes dimensiones:

Primera dimensión: Gastos corrientes, indica el MEF (2020) que son los desembolsos de conservación e intervención de los servicios prestados por el país. Para incidir en la clasificación económica, considera que la clasificación general de gastos es la misma que los que se convertirán en indicadores.

Segunda dimensión: Gastos de capital, hace mención el MEF (2020) que son destinados a incrementar el rendimiento o el patrimonio nacional de forma inmediata o futura. Para el impacto del a clasificación económica, considera los gastos generales por adquisición de activos no financieros.

Tercera dimensión: Categoría presupuestal, menciona el MEF (2016), "Es un estándar para clasificar los gastos presupuestarios. Esto como parte de una estructura programática”. 
Para la segunda variable, calidad de gasto, Bastidas et al. (2016), "La calidad del gasto contiene los elementos que dan garantía usar de manera eficaz y eficiente los recursos del estado, con la finalidad de dar crecimiento al desarrollo económico" (p.3). Así también la Directiva 002-2016-EF-50.01 - Directiva para los programas presupuestales en el marco del presupuesto por resultados, en el anexo 1, inciso a) hace mención que "La calidad como la medición de capacidades en la intervención para dar respuesta a las intervenciones con la población en su conjunto" (p.14). Por tanto, se debe priorizar a la mejorar de los procedimientos de acuerdo a las siguientes dimensiones:

Primera dimensión: Eficiencia, MEF (2016), “Éste busca el rendimiento de los recursos empleados y la conexión al producir un bien o servicio entre los insumos que emplean para su producción” (p.40).

Segunda dimensión: Eficacia, MEF (2016), "Cuantifica el nivel de que se cumplan con los fines establecidos por la entidad" (p.40).

Tercera dimensión: Economía, MEF (2016), "Mensura la suficiencia de disponer apropiadamente los recursos financieros" (p.40). Además, Sevilla Arias (2015) indica que estudia cómo administrar los recursos aptos para saciar las necesidades que la ciudadanía tiene.

\section{PROCEDIMIENTOS METODOLÓGICOS}

El presente estudio fue de enfoque cuantitativo, como lo menciona Hernandez et al. (2003) indica que este tipo de enfoque recolecta datos para comprobar la hipótesis como mediciones numéricas y estadísticas (p. 4). Siendo de tipo aplicada, Basándonos en que se considera llegar a resolver un problema que ha sido encontrado como indica CONCYTEC (2018), la investigación es de tipo aplicada, se utilizó un enfoque cuantitativo, porque tiene como objetivo resolver problemas concretos y prácticos planteados, probando la hipótesis planteada en la presente investigación con mediciones numéricas y haciendo uso de la estadística. El diseño que se empleo es no experimental, tal como señala Hernández et al. (2003), "Es transversal, de modo que se tuvo un plazo determinado para realizarse, también fue descriptivo y relevante, porque se dispuso la existencia del vínculo entre las variables estudiadas, como la ejecución presupuestaria y la calidad del gasto" (p.151). Dentro de la variable ejecución presupuestal tenemos las siguientes dimensiones: Gastos corrientes, gastos de capital, categoría presupuestal y la variable calidad de gasto con las dimensiones: Eficiencia, eficacia y economía. La población que de acuerdo a Maldonado Pinto (2018) afirma que la población es una agrupación de recursos que incluye personas, cosas, objetos, documentos, los cuales incorporan y forman parte de un problema de indagación, tiene la cualidad de ser estudiada, medida y cuantificada (p.145). está se encuentra compuesta por todos los trabajadores de la entidad y reportes de ejecución presupuestal. La técnica utilizada para recopilar datos, fue la encuesta que indica López y Fachelli (2015) "Es una técnica de recolección de datos mediante la interrogación a los individuos, realizada por el cuestionario y administrado de forma protocolaria, característico por el anonimato del sujeto" (p.14). y el análisis documental se "Constituye un total de operaciones eruditas, que buscan describir y presentar documentos de forma sistemática y unificada para posibilitar su recuperación, abarca análisis y procesamiento integral, que a su vez incluye bibliografía y descripción general" (Clauso García, 1993, p.1). Los instrumentos utilizados son: cuestionario que se ha encontrado separadas por dimensiones e indicadores y guía de análisis documental se procesa la información presupuestal obtenida del Sistema Integrado de Información Financiera del Sector Publico SIAF-SP, para la validación de los mismos fue anticipadamente verificado y validado mediante dictamen de expertos, por profesionales con conocimiento del tema de investigación. El análisis de confiabilidad del instrumento se determinó a través del análisis del Alfa de Cronbach de acuerdo a Oviedo y Campo-Arias (2005) indican que el alfa de Cronbach es el promedio de las correlaciones entre los ítems que hacen parte de un instrumento, donde se obtuvo un valor de 0.907 para la variable ejecución presupuestal y 0.892 para la variable calidad de gasto. El procedimiento para obtener la normalidad fue el test de Shapiro-Wilks, debido a que se aplican a muestras menores a 50, obteniendo en esta una normalidad por lo que se aplicó la prueba de correlación de Pearson como lo menciona Ruiz (s.f) que esta se utiliza para estudiar la relación entre dos variables aleatorias o normales cuantitativas (par. 4).

\section{RESULTADOS Y DISCUSION}

Determinar la relación que existe entre ejecución presupuestal y calidad de gasto de la Municipalidad Distrital de Shapaja, periodo 2019 
Tabla 2

Relación entre ejecución presupuestal y calidad de gasto de la Municipalidad Distrital de Shapaja, periodo 2019

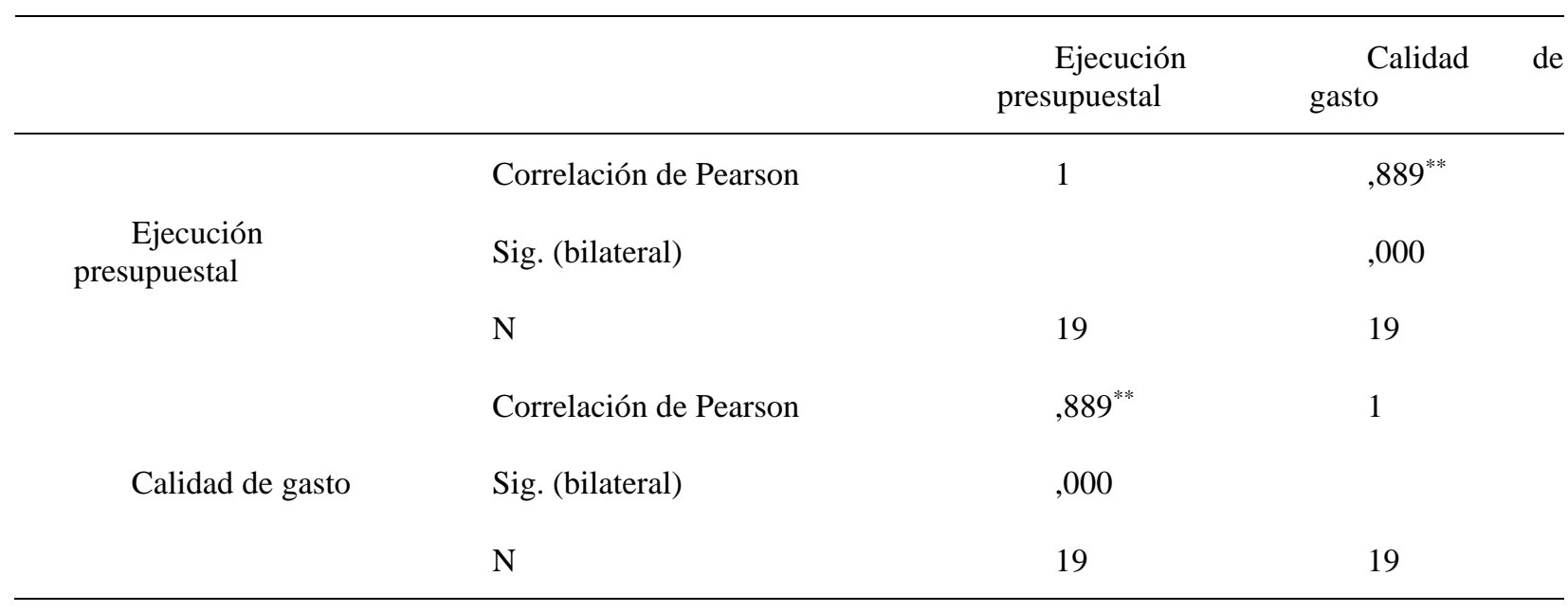

**. La correlación es significativa en el nivel 0,01 (bilateral).

Nota. La correlación de Pearson entre ejecución presupuestal y calidad de gasto de la Municipalidad Distrital de Shapaja, periodo 2019 es alta; con valor de (0.889) y la significancia bilateral menor a 0,05 ( $p<0,05)$. Por tanto, mejorar la ejecución presupuestal relacionado a los gastos corrientes, gastos de capital y categoría presupuestal abonanzará la calidad de gasto en todas sus dimensiones, tal como se puede apreciar en la tabla 13. 2019

Determinar la relación del gasto corriente y calidad de gasto de la Municipalidad Distrital de Shapaja, periodo

Tabla 3

Relación entre el gasto corriente y calidad de gasto de la Municipalidad Distrital de Shapaja, periodo 2019

\begin{tabular}{|c|c|c|c|}
\hline & & $\begin{array}{l}\text { Gasto } \\
\text { corriente }\end{array}$ & $\begin{array}{l}\text { Calidad } \\
\text { gasto }\end{array}$ \\
\hline \multirow{3}{*}{ Gasto corriente } & Correlación de Pearson & 1 &, $680^{* *}$ \\
\hline & Sig. (bilateral) & & ,001 \\
\hline & $\mathrm{N}$ & 19 & 19 \\
\hline \multirow{3}{*}{ Calidad de gasto } & Correlación de Pearson &, $680^{* *}$ & 1 \\
\hline & Sig. (bilateral) &, 001 & \\
\hline & $\mathrm{N}$ & 19 & 19 \\
\hline
\end{tabular}

\footnotetext{
**. La correlación es significativa en el nivel 0,01 (bilateral).
}

Nota. Coexiste relación entre el gasto corriente y calidad de gasto de la Municipalidad Distrital de Shapaja, periodo 2019, con correlación de Pearson $(r=680)$ y la significancia bilateral menor a 0,05 $(p<0,05)$. Es preciso indicar que, al mejorar los gastos corrientes orientado al personal y obligaciones sociales, además de las pensiones y otras prestaciones sociales, al realizar acciones de mejoras en los servicios básicos de la comunidad por el presupuesto asignado para la adquisición de Bienes y Servicios y la contratación acorde a la programación inicial, contribuiría a dar mejora a la calidad de gasto en la institución.

Determinar la relación del gasto de capital y calidad de gasto de la Municipalidad Distrital de Shapaja, periodo 2019

\section{Tabla 4}

Gasto de capital y calidad de gasto de la Municipalidad Distrital de Shapaja, periodo 2019 


\begin{tabular}{llll}
\hline & & \multicolumn{1}{c}{$\begin{array}{c}\text { Gasto } \\
\text { capital }\end{array}$} & $\begin{array}{c}\text { Calidad de } \\
\text { gasto }\end{array}$ \\
\hline Casto capital & Sig. (bilateral) & 1 &, $684^{* *}$ \\
& $\mathrm{~N}$ & 19 &, 001 \\
& Correlación de Pearson &, $684^{* *}$ & 1 \\
$\begin{array}{c}\text { Calidad de } \\
\text { gasto }\end{array}$ & Sig. (bilateral) &, 001 & 19 \\
& $\mathrm{~N}$ & 19 & 19 \\
\hline
\end{tabular}

**. La correlación es significativa en el nivel 0,01 (bilateral).

Nota. Existe relación entre gasto capital y calidad de gasto de la Municipalidad Distrital de Shapaja, periodo 2019, con correlación Pearson $(r=684)$, siendo esto una correlación moderada y la significancia bilateral menor a 0,05 ( $p<0,05)$. Por tanto, al realizar estrategias relacionados a la mejora de la ejecución del presupuesto programado para estudios de pre inversión, ejecuciones físicas por la ejecución de obras y proyectos, esto impactará en gran medida en la calidad de gasto.

Determinar relación de la categoría presupuestal y calidad de gasto de la Municipalidad Distrital de Shapaja, periodo 2019

Tabla 5

Categoría presupuestal y calidad de gasto de la Municipalidad Distrital de Shapaja, periodo 2019

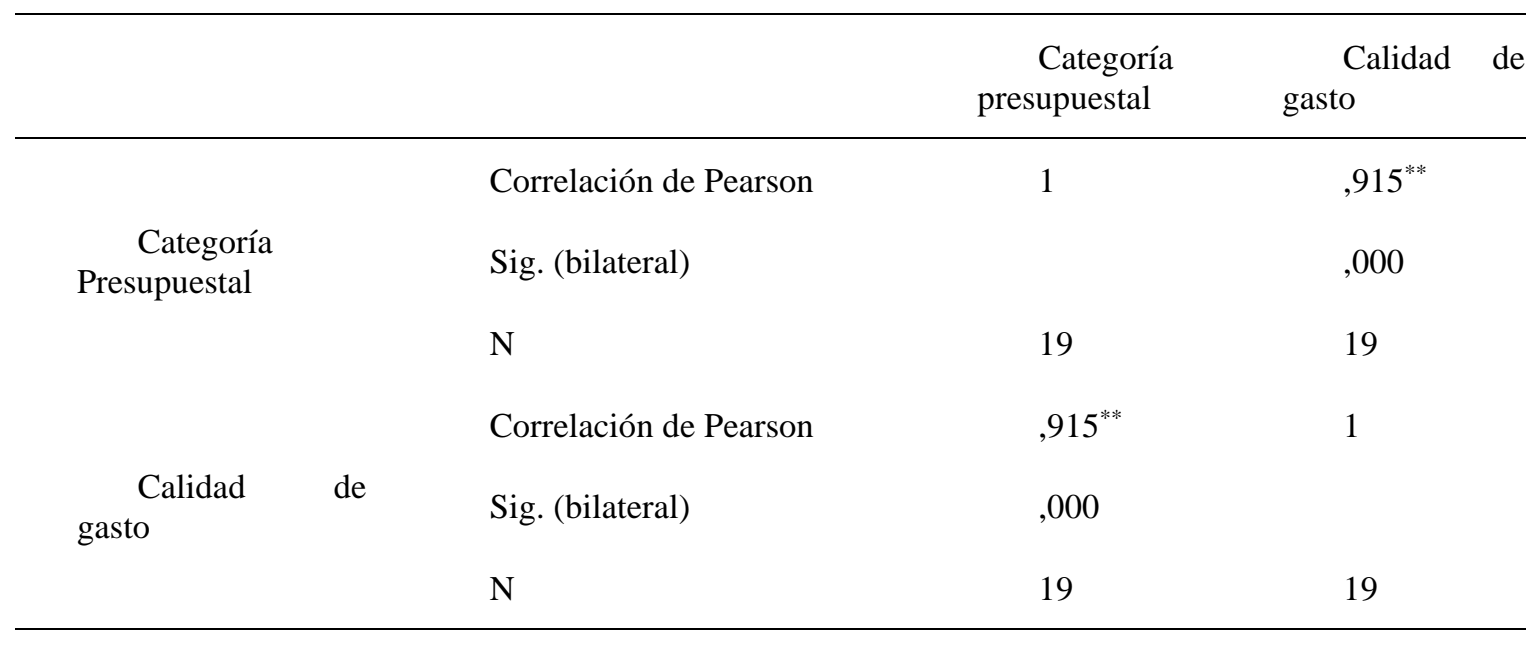

**. La correlación es significativa en el nivel 0,01 (bilateral).

Nota. Existe relación de la categoría presupuestal y calidad de gasto de la Municipalidad Distrital de Shapaja, periodo 2019, con correlación de Pearson $(r=0,915)$ y el nivel de significancia es de 0,00 $(p<0,05)$, lo cual significa una relación entre las variables. Por tanto, al mejorar las estrategias de la categoría presupuestal relacionadas al recojo de residuos sólidos por parte la entidad, abastecimiento de agua potable y/o agua entubada, obtención de una buena gestión con la correcta ejecución de su presupuesto, mejoramiento de recaudación de los ingresos propios (Recursos Directamente Recaudados e Impuestos Municipales, incremento de cartera de perfiles y estudios de pre inversión) mejorará la calidad del gasto.

\section{CONCLUSION}

Se concluye que hay una conexión directa entre la ejecución presupuestal y la calidad de gasto de la Municipalidad Distrital de Shapaja. Esto por medio del procesamiento de datos estadísticos con una significancia bilateral $(\mathrm{p}=0.000)$. La intensidad de relación fue positiva con 0,889 de coeficiente de correlación. Esto significa que las respuestas obtenidas de que estas variables se proporcionan. 
Las limitaciones de la presente investigación estuvieron relacionadas con la entrega de información debido a que muchas entidades consideran que es material confidencial, obstaculizando así de esta manera el acceso rápido a la información, así mismo se tuvo problemas para la aplicación del cuestionario como instrumento de recolección de datos, ya que debido a la coyuntura nacional a causa de la Covid 19, no ha sido posible establecer relación directa con la población en estudio.

Se sugiere la realización de estudios cualitativos, utilizando otras medidas relacionadas al objeto del estudio y hacer posibles comparaciones sobre los resultados obtenidos en periodos y contextos diferentes.

\section{REFERENCIAS}

Barona, L. (2019). El plan operativo anual y la ejecución presupuestaria de las universidades y escuelas politécnicas públicas de la zona 3. [Tesis de Maestría, Universidad Técnica de Ambato]. https://repositorio.uta.edu.ec/bitstream/123456789/29136/1/T4416M.pdf

Bastidas, C., Andocilla J., Franco W. (2016). Consideraciones sobre la calidad del gasto público. Revista. Revista Publicando. https://revistapublicando.org/revista/index.php/crv/article/view/284/pdf_155

Campoverde, R. \& Pincay, D. (2019). Ejecución Presupuestaria vs Rendición de Cuentas, sobre el rubro de Investigación: Caso de una Universidad Ecuatoriana (Artículo Científico) Digital Publisher, Ecuador. https://dialnet.unirioja.es/descarga/articulo/7144023.pdf

Chafloque, C. (2017). El presupuesto por resultados y sus efectos en la calidad del gasto del sector salud de la Región Lambayeque 20112016 [Tesis de Maestría, Universidad Cesar Vallejo] https://hdl.handle.net/20.500.12692/8410

Clauso, A. (1993). Análisis Documental: el análisis formal. (Revista) Revista General de información y documentación, España. https://revistas.ucm.es/index.php/RGID/article/view/RGID9393120011A/11739

CONCYTEC (2018). Compendio de normas para trabajos escritos. Colombia: Instituto Colombiano de Normas Técnicas y Certificación, CONCYTEC.

Economía y Finanzas, M. (2016). Directiva Nº006-2016-EF/50.01Directiva para la programación y formulación anual del presupuesto del sector público, con una perspectiva de programación multianual Perú: Diario el Peruano. https://www.mef.gob.pe/contenidos/archivosdescarga/TUO_Directiva_Programacion_Multianual_RD006_2016EF5001.pdf

Economía y Finanzas, M. (2021). Directiva No 0001-2021-EF/50.01 - Directiva de Programación Multianual Presupuestaria y ... Ley de Presupuesto del Sector Público para el Año Fiscal 2021: Diario el Peruano. https://www.mef.gob.pe/es/normatividad-sp-9867/por-instrumento/directivas/24954-directiva-n-0001-2021-ef50-01/file

Economía y Finanzas, M. (2020). Directiva para la Ejecución Presupuestaria. Perú: Diario el Peruano. https://cdn.www.gob.pe/uploads/document/file/1510868/RD0034_2020EF5001.pdf.pdf

El peruano. (2019). Aprueban los procedimientos para el cumplimiento de metas y la asignación de recursos del Programa de Incentivos a la mejora de la gestión municipal del año 2021, y dictan otras medidas. Lima: Diario Oficial del Bicentenario. https://busquedas.elperuano.pe/normaslegales/aprueban-los-procedimientos-para-elcumplimiento-de-metas-y-decreto-supremo-n-397-2020-ef-1914061-1/

Engl, R., Dineiger, P. \& Garcia, M. (2016). Fiscalización y calidad del gasto público en América Latina y el Caribe. (Libro) Cooperación Alemana, Alemania. https://www.olacefs.com/wp-content/uploads/2017/04/Calidad-delgasto-publico-WEB.pdf

EPG Universidad Continental (11/1/2018). Aclarando conceptos: eficacia vs. eficiencia. Blog Posgrado. https://blogposgrado.ucontinental.edu.pe/aclarando-conceptos-eficacia-vs-eficiencia

Fernanda Viante, R., Suave, R., \& da Silva Mafra, M. (2021). Execução do Orçamento em Santa Catarina: Evolução das Despesas de 2006 A 2018. Revista FSA, 18(2), 108-127. https://doi.org/10.12819/2021.18.02.6

García, AL. (2002). Tratamiento y análisis de la documentación. En: Vizcaya Alonso, D. (comp). Selección de lecturas: Fundamentos de la organización de la información. La Habana: Universidad de La Habana.

http://scielo.sld.cu/scieloOrg/php/reflinks.php?refpid=S1024-

9435200400020001100001\&lng=es\&pid=S1024-94352004000200011

Hernández, S., Fernández, C. y Baptista, L. (2014). Selección de la muestra. En Metodología de la Investigación (6ª ed., pp. 170-191). México: McGraw-Hill. http://observatorio.epacartagena.gov.co/wpcontent/uploads/2017/08/metodologia-de-la-investigacion-sexta-edicion.compressed.pdf

Informe El Comercio-IPE, (2 de diciembre de 2019). Presupuesto 2020 bordea los S/177.000 millones: ¿Cómo reducir el gasto público ineficiente? El Comercio, Perú. https://elcomercio.pe/economia/peru/presupuesto-2020bordea-los-s177000-millones-como-reducir-el-gasto-publico-ineficiente-noticia/

Lemus, D., Torres, A., Cubillos, S. \& Camelo, F. (2017). Análisis de la ejecución presupuestal en Colombia (Artículo Científico) Revista CIFE 30, Colombia. https://dialnet.unirioja.es/descarga/articulo/6852525.pdf 
Ley 28411. (2004). Ley General del Sistema Nacional de Presupuesto. Perú. http://transportes.junin.gob.pe/main.php/ver_documento/id /drtc0fc8dfdca9f9e54c9ccddc663e514203.pdf/

Ley Marco de la Administración Financiera del Sector Público - Ley No 28112 https://www.mef.gob.pe/es/normatividad-sp-9867/por-tema/normativa-basica-presupuestaria/7343-ley-n28112-3/file

López, P. \& Fachelli, S. (2015). Metodologia de la investigacion social cuantitativa. Libro. Universitat Autònoma de Barcelona. España. 1a Ed. https://ddd.uab.cat/pub/caplli/2016/163567/metinvsoccua_a2016_cap2-3.pdf

Luna, A. (2016). La evaluación por calidad del gasto y su incidencia en la adecuada ejecución presupuestaria en la Dirección Regional de Salud Cajamarca, Período 2014 (Tesis) Universidad Nacional de Trujillo, Perú. https://1library.co/document/dzxk9mvq-evaluacion-incidencia-ejecucion-presupuestaria-direccion-regionalcajamarca-periodo.html

Maldonado, J. (2018). Metodología de la investigación Social. Cuantitativo, socio crítico, cualitativo, complementario. ( $\left.1^{\circ} \mathrm{ed}\right)$. Colombia. Ediciones de la U. https://es.scribd.com/read/436222099/Metodologia-de-la-investigacionsocial-Paradigmas-cuantitativo-sociocritico-cualitativo-complementario

Mina, K. (2016). Los ingresos tributarios y su incidencia en el presupuesto del gobierno autónomo descentralizado municipal del cantón Esmeraldas, período 2012-2015 (Tesis de Maestría) Universidad de Guayaquil, Ecuador. http://repositorio.ug.edu.ec/handle/redug/13927

Morales, J., Coyla M., Vargas N., Ramos J., Quispe D. , Huanca J. (2018). Level of Budget Execution According to the Professional Profile of Regional Governors Applying Machine Learning Models 2020 International Journal of Advanced Computer Science and Applications https://thesai.org/Downloads/Volume11No11/Paper_39Level_of_Budget_Execution.pdf

Murillo, W. (2008). La investigación científica. http//www.monografias.com/trabajos15/investcientífica/investcientífica.shtm

Santiago, N. (2018). Formulación de Presupuestos (Revista) Universidad Técnica de Ambato, Ecuador. https://revistas.uta.edu.ec/Books/libros\%202019/presupuesto.pdf

Olivera, E. (2018). Ejecución Presupuestal de Inversiones y su Influencia en el logro de metas de la Municipalidad Provincial de Cutervo 2017 [Tesis de pregrado, Universidad Cesar Vallejo] https://hdl.handle.net/20.500.12692/27651

Omonte, J. y Rojas, V. (2019). Proceso de ejecución presupuestaria y la calidad de gasto en una unidad ejecutora de una entidad del estado, Lima 2019 [Tesis de grado, Universidad San Ignacio de Loyola] http://repositorio.usil.edu.pe/handle/USIL/9655

Otzen, T. \& Manterola, C. (2017). Técnicas de Muestreo sobre una Población a Estudio. International Journal of Morphology, 35(1), 227-232. https://dx.doi.org/10.4067/S0717-95022017000100037

Oviedo, H. \& Campo-Arias, A. (2005). Aproximación al uso del coeficiente alfa de Cronbach. Revista Colombiana de Psiquiatría, 2005, vol.34, n.4, pp.572-580. ISSN 0034-7450. http://www.scielo.org.co/pdf/rcp/v34n4/v34n4a09.pdf

Paredes, L. (2020). Ejecución presupuestal y calidad del gasto público en la Municipalidad Distrital de Shamboyacu 2019 [Tesis Posgrado, Universidad Cesar Vallejo] https://repositorio.ucv.edu.pe/bitstream/handle/20.500.12692/48385/Paredes_ULSD.pdf?sequence $=1 \&$ is Allowed $=y$.

Paco, L. y Mantari, W. (2014). El proceso de ejecución presupuestal y su influencia en la calidad de gasto de la municipalidad provincial de Huancavelica periodo 2013" [Tesis de pregrado, Universidad Nacional de Huancavelica] https://docplayer.es/139462508-Universidad-nacional-de-huancavelica.html

Patiño, L. (2017). Influencia del presupuesto por resultados en la calidad del gasto público del Municipio de Medellín. Caso del sector de la vivienda, en el periodo 2008-2016 [Tesis de Maestría, Universidad EAFIT]. https://repository.eafit.edu.co/bitstream/handle/10784/11733/Pati\%c3\%b1oMu\%c3\%b1oz_LauraAndrea_2017 .pdf?sequence $=1 \&$ isAllowed $=\mathrm{y}$

Quispe, M. (2016). Influencia del presupuesto por resultados y su incidencia en la gestión del gasto de la Municipalidad Distrital de Atuncolla, periodos 2013 - 2014 [Tesis de grado, Universidad Nacional del Altiplano] http://repositorio.unap.edu.pe/bitstream/handle/UNAP/5250/Quispe_Apaza_Mariluz.pdf?sequence=1\&isAllow $\mathrm{ed}=\mathrm{y}$

Sevilla, A. (2015). Economía. Economiedia.com https://economipedia.com/definiciones/economia.html

Toledo, Y. (2017). Análisis de la Gestión en la Ejecución Presupuestaria en la Corporación Eléctrica del Ecuador CELEC EP Unidad de Negocio Termoesmeraldas. [Tesis de Maestría, Pontificia Universidad Católica de Ecuador]. https://repositorio.pucese.edu.ec/handle/123456789/1066

Yactayo, E. (2019). La ejecución presupuestal de ESSALUD del Perú como un instrumento de gestión. (Artículo Científico). Pensamiento Crítico de la Facultad de Ciencias Económicas, Universidad Nacional Mayor de San Marcos. Perú. https://revistasinvestigacion.unmsm.edu.pe/index.php/econo/article/download/1656 
Rodríguez, M., Palomino, G., \& Aguilar, C. (2020). Eficiencia, eficacia y transparencia del gasto público municipal. Ciencia Latina Revista Científica Multidisciplinar, 4(2), 704-719. https://doi.org/10.37811/cl_rcm.v4i2.108

Ruiz, L. (s/f). Coeficiente de correlación de Pearson: qué es y cómo se usa. Psicologia y Mente. https://psicologiaymente.com/miscelanea/coeficiente-correlacion-pearson

Todesco, C., \& Cardoso da Silva, R. (2021). Planejamento setorial e execução orçamentária em turismo no Brasil (2003-2018). Revista Brasileira de Pesquisa Em Turismo, 15(2), 1-19. https://doi.org/10.7784/rbtur.v15i2.1986

Torres, A., Cubillos, J., Camelo, F., \& Lemus, D. (2018). Análisis de la ejecución presupuestal en colombia durante el periodo 1954 - 2013. Revista CIFE: Lecturas De Economía Social, 19(30), 23-45. https://doi.org/10.15332/22484914/3888

Zapata, L., Chávez J. (2018). Las modificaciones presupuestarias en el marco de los programas presupuestales. Revista. Administración Pública \& Control N48/ Diciembre 2017. 